\title{
Public engagement with science: ways of thinking and practicing
}

\author{
Elizabeth Stevenson \\ School of Chemistry \\ University of Edinburgh \\ Edinburgh \\ EH9 3JJ
}

e.stevenson@ed.ac.uk
Public engagement enables the sharing of knowledge with a wider, non-specialist community, regardless of the level of previous knowledge of the community...

\begin{abstract}
The primary focus of the Higher Education Institution (HEI) is the generation and dissemination of knowledge. This knowledge is generated and shared throughout the research community and to students specifically enrolled in university programmes. Public engagement with science enables and ensures the generation and sharing of knowledge throughout a wider community.
\end{abstract}

Public engagement with science has enjoyed an increasingly heightened profile in recent years with six 'Beacons for Public Engagement" being established across HEls in the UK, including a National Co-ordinating Centre for Public Engagement ${ }^{2}$ hosted between the University of Bristol and the University of the West of England In addition, public engagement is a component in the 'Pathways to Impact' statements ${ }^{3}$ which have been introduced into all RCUK research funding applications.

However public engagement, and in particular public engagement with science, can often be perceived as an add-on or 'Cinderella' activity to be undertaken only by the dedicated and often only in their own time. This paper argues that public engagement with science is a legitimate area of academic practice in HEls which complements and extends research and teaching. The paper outlines key principles which underpin public engagement with science and describes effective work practice.

\section{Introduction}

Higher Education institutions are establishments in which knowledge is generated and disseminated. Yet that knowledge often remains 'hidden' within the confines of the academic community. This creates the phenomenon of the 'ivory tower' in which the knowledge generated by a university remains within the academic community and can even remain within individual disciplines. It could be said that, in some instances, the ivory towers are created from the inside by the academic community themselves.

Public engagement enables the sharing of knowledge with a wider, non-specialist community, regardless of the level of previous knowledge of the community and, as a practice, can enable the enrichment and understanding of knowledge by viewing it from different perspectives. Public engagement involves extending the reach of and engagement with a discipline both within and beyond the HEl (Figure 1). Therefore public engagement with science is an extension of the knowledge generation and dissemination of an $\mathrm{HEI}$ and lies on a continuum (in the dissemination of knowledge of an $\mathrm{HEI}$ ) rather than being a completely separate entity.

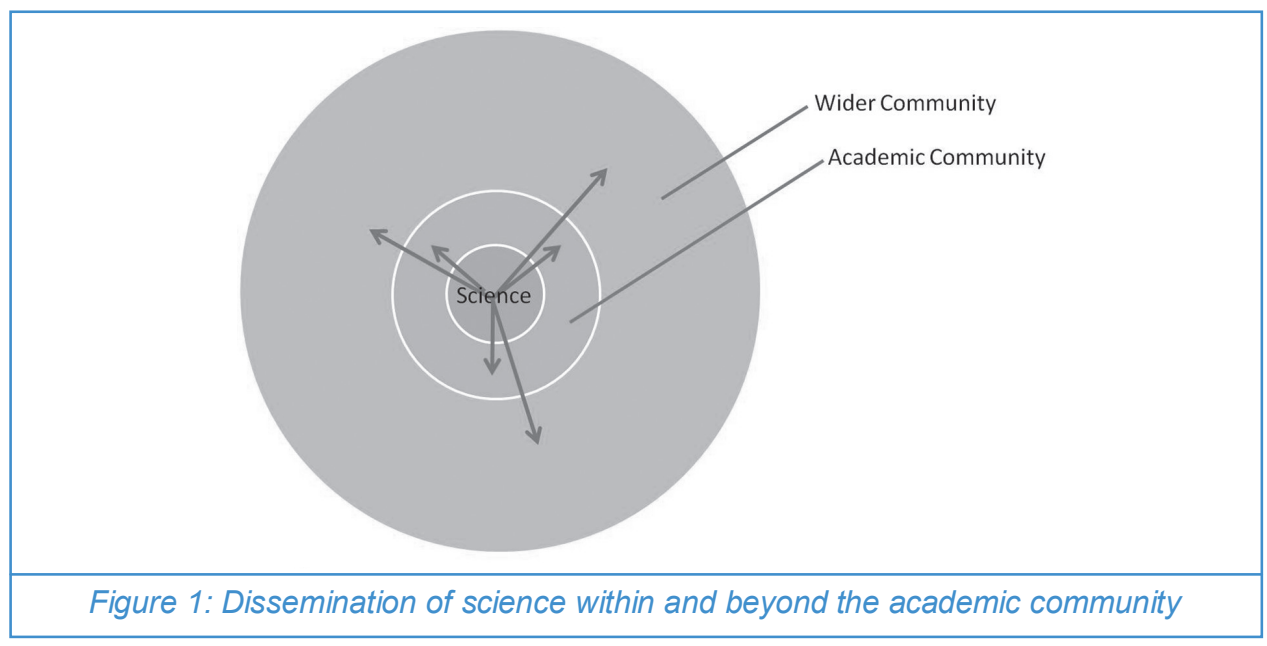


The UK Higher Education Funding Councils, Research Funding Councils UK, and the Wellcome Trust have recognised the importance and value of embedding public engagement as a practice in higher education and have established the Beacons for Public Engagement initiative: a four-year project designed to create a culture change across the higher education sector. The Beacons initiative supports six Beacons for Public Engagement across the UK, together with a National Co-ordinating Centre for Public Engagement (NCCPE). ${ }^{2}$

The Beacons project is unique as a culture change project in several aspects:

- The focus of the project is to establish a culture of public engagement across all disciplines in HEls in the UK. HEls who are not directly involved with any of the six Beacons have access to the knowledge and tools for culture change being generated by the NCCPE ${ }^{4}$.

- The project involves the HEI community at all levels e.g. researchers, public engagement practitioners, Heads of Schools, Vice Principals.

- The project is engaging the HEI community in a process of participatory research ${ }^{5}$ in order to generate and refine learning about public engagement, to define public engagement and thereby to embed the practice as a legitimate area of work in HEls.

- Having defined the key purposes for public engagement i.e. informing, consulting and collaborating ${ }^{6}$, the Beacons project has employed these ' ways of working' i.e. (informing, consulting and collaborating) in their journey to establish a culture of public engagement in HEls. The Beacons are "practicing what they preach" says Heather Rea, Project Manager of Edinburgh Beltane, Beacon for Public Engagement.

- Each of the six Beacons, established across the UK, has a different focus. This allows for experimentation in working towards culture change and recognises that different approaches to culture change depend on existing cultures in HEls and the communities and publics with which they engage.
The focus of the Beacons project is to establish a culture of public engagement practice across all disciplines within an $\mathrm{HEI}$. The three main purposes for public engagement as defined by NCCPE are informing, consulting and collaborating. However much of the public engagement with the physical sciences, which is practiced by HEls, is for the purpose of informing or sharing knowledge: knowledge about key concepts, research findings, potential applications of research or about the research process and the scientific method. Knowledge which is generated by this process of public engagement with science may not be scientific knowledge. It is more likely to be insights regarding the application of the science, insights regarding methodologies for dissemination and insights about misunderstandings of scientific concepts.

Public engagement with science as a 'way of thinking'. How can one communicate a piece of research or indeed a fundamental scientific concept to a non-specialist audience when it has taken the researcher or scholar many years of study and research to develop the research, and to arrive at the understanding themselves? A different approach is required in order to convey the knowledge, one which does not depend on the audience having been immersed in the discipline and therefore having benefited from the iterative effect of years of gaining knowledge in the discipline. This 'way of thinking' involves viewing the science in the wider context i.e. from a 'bigger picture' perspective. The public engagement with science approach is to view the science from the outside in, rather than from the inside out, taking a step back (or several steps back from the detail) and finding a point or points of common interest with the 'audience'.

Figure 2 shows:

a. shows a photograph of the centre of a sunflower. This is analogous to the level of detail in a research paper.

b. shows the view of a whole sunflower, and illustrates the process of stepping back from the detail of the research paper to view the science in context to enable finding the points of common interest with the audience.

c. shows a whole field of sunflowers, steps back even further to a much wider context.

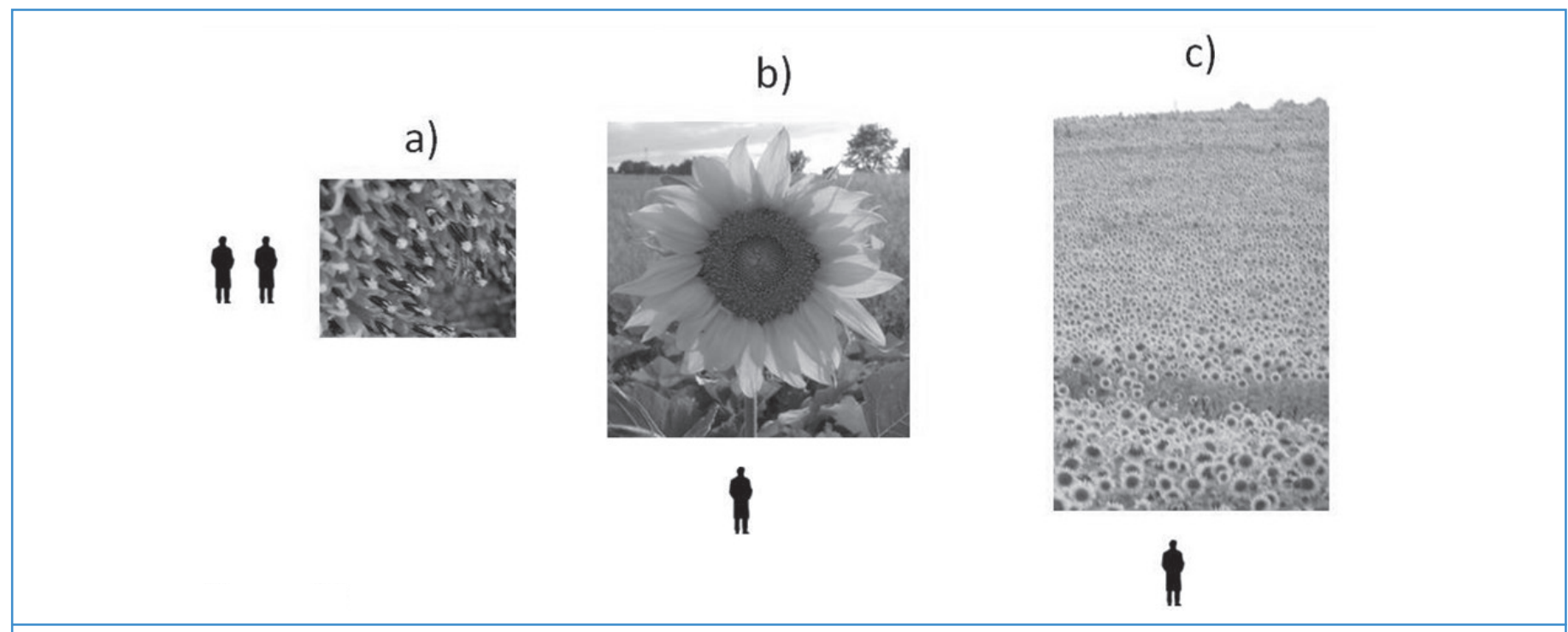

Figure 2: Viewing the science from a wider perspective 
In public engagement with science, it is important to choose an appropriate level and 'hook' for both the audience and the purpose of the engagement.

For example, my chemistry research was in Pyrolitic Syntheses of Fused Bridgehead Nitrogen Heterocycles ${ }^{7}$. This description is at an appropriate level for a PhD thesis and for research papers.

Taking a step back, I describe the work as follows: selecting a starting material (i.e. chemical compound) and heating it under vacuum to temperatures of up to $1000^{\circ} \mathrm{C}$. This enables rearrangement (by bonds breaking and reforming in a different configuration) of the original starting material to a different compound. Taking a further step back I use an analogy i.e. the Molecular Anagram. Taking a carefully chosen word (molecule) rearranging the letters (atoms) to form a different word (molecule). The analogy can be extended: sometimes the rearrangement leads to another word (molecule) sometimes leads to nonsense (or in the case of my research, tar!).

Another example is drawn from the staff profiles on the University of Edinburgh, School of Chemistry web-page describing the work of Philip Camp ${ }^{8}$.

"We study the properties of complex fluids e.g. colloidal suspensions, ferrofluids. Using computational and theoretical techniques, we determine the connections between the structure, dynamics, and phase behaviour of complex fluids, and the properties of the constituent molecules. Then we construct simple molecular models that capture the essential characteristics of real systems and study these models using computational techniques." The above description does not contain the level of detail of a research paper, however it does capture the essence of the work accurately and for a scientific audience.

Taking a further step back and for a non-scientist audience, Philip Camp said the following about his and others' work: "Researchers in the School of Chemistry use computer movies to give them amazing insights on the atomic world."

Taking several steps back from the level of details of the research paper enabled the researchers to describe the key focus of the research in a single sentence. This 'way of thinking' is invaluable within the research community. As multidisciplinary projects become the norm, it is essential that researchers from one discipline can communicate effectively with another to deliver research projects and to explore exciting new avenues of research. This requires stepping back from the level of detail of the research paper in a discipline and exploring the research area or phenomenon at the appropriate level at which communication is productive and conducive to developing a research area.

This 'way of thinking' is necessary when considering 'Impact' of research: in the composition of 'Pathways to Impact' statements now required in funding applications for RCUK and for the Impact Case Studies which will be required for the Research Excellence Framework ${ }^{9}$. The questions to be answered are as follows: Where does the research sit within a wider context, who will benefit and how will they benefit? Impact can be thought of as a tool to assist in the strategic planning of research.
Key principles of public engagement with science. This 'wider context' approach, when combined with imagination, creativity and lateral thinking are essential in the development of an activity for public engagement. There is a huge range of methodologies and formats employed in public engagement: from the demonstration lecture, interactive exhibits and hands-on workshops through to novel examples such as chemistry comics ${ }^{10}$ and maths walks ${ }^{11}$.

However, underpinning effective public engagement with science are key guiding principles and practice which are common to all methodologies and formats:

- The science should be accurately represented (the level at which the concept is communicated will not be that of a research paper however the level, analogies used, and methodologies should convey the science accurately).

- The activity should be considered and designed from the audience perspective (i.e. what may be fascinating to a scientist may not be the 'hook' for the non-specialist audience).

- There should be clarity of purpose for the engagement e.g. are you seeking to generate interest, inform, provide a practical experience, clarify understanding, seek information or views

- The experience should be neutral: public engagement is not PR.

"As we understand 'engagement' to require active involvement and mutual benefit it is possible to also draw a line and to exclude certain types of interactions with the public: for instance, PR campaigns, which seek to persuade the public of a particular point of view" ${ }^{12}$.

- The engagement experience should aim to produce an enriching experience for the participants. The participants include both the engager and the 'engagee'. This enrichment may be knowledge gained, deeper insight, a different way of looking at or doing something. This is beautifully summed up in a statement by Monty Don ${ }^{13}$ He was referring specifically to gardens, which could be construed as a form of public engagement, however his statement is relevant to all successful public engagement: "It enlarges us". We should come out (of the experience) "with a whole new set of parameters with which to measure life".

How does one work towards achieving this effect when planning a public engagement experience? Figure $3^{14}$ presents an invaluable tool for designing and evaluating a public engagement activity:

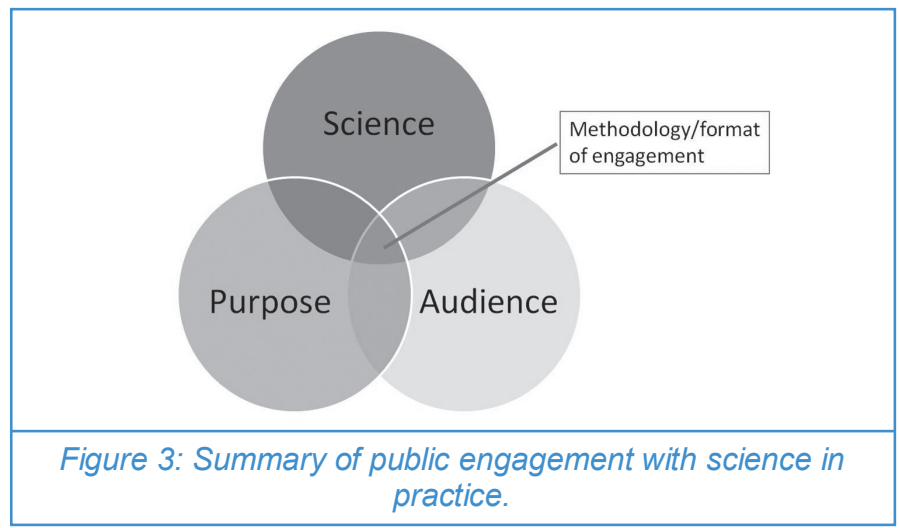




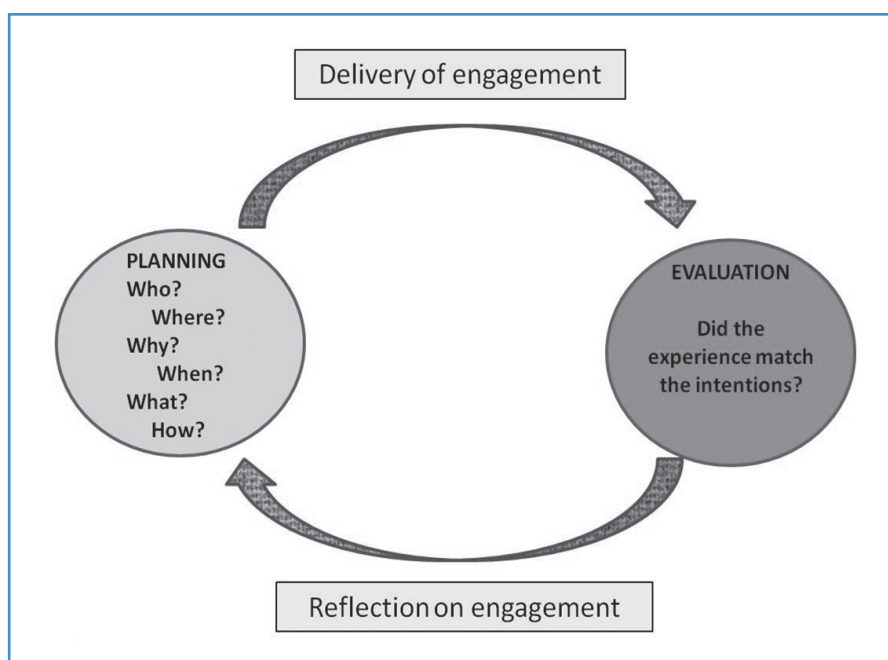

Figure 4: Reflective practice in public engagement with science

Note that the science, audience and purpose of the engagement require equal consideration and ideally the methodology for the public engagement should fall within the overlap of these sectors. By considering the audience and the purpose of the engagement from the outset, the practitioner can establish realistic objectives and outcomes for the activity or project and build in an effective evaluation strategy at the planning stage.

\section{'Ways of practicing' in public engagement with science.}

In addition to the key principles, there are 'ways of practicing' in public engagement with science which are common to all effective science engagement activities:

- Reflective practice

- Professionalism

- Flexibility

- Quid pro quo

Public engagement is very much a 'practice based' area of work in which learning for the practitioner is acquired through practice, rather than through theory. However, reflective practice is essential for effective development as a practitioner in public engagement as indeed it is for other areas of work including teaching.

By asking the questions (who, where, why, when, what and how) during the design and development of an engagement activity enables the practitioner to identify and access the knowledge, experience and support required for the activity. By having a clear idea of the purpose of the activity the practitioner can then evaluate the effectiveness of the activity by comparing the experience with the intention. The insights gained from this process inform subsequent engagement ${ }^{15}$. Reflection in practice ${ }^{16}$ is also vitally important in public engagement, particularly when there is direct interaction between the engager and the audience. A personal maxim of mine is that in any face-to-face public engagement activity: 'Something unexpected will occur! You won't know what it is until it happens but you can be sure it will happen'. Reflection in practice, professionalism and flexibility are critical in these circumstances. Being able to adapt the engagement experience in real time requires good awareness, problem-solving ability, imagination, creativity and experience.
Rarely do you have the luxury of the perfect venue, a homogenous audience or perfectly aligned expectations, so an ability to reflect and act in practice, to behave professionally and to be flexible and creative in resolving issues is essential. These are key transferable skills, some of which are identified in the Chemistry Benchmarking Document ${ }^{17}$

Let's not forget that the unexpected can be a challenge to address or it can be a completely unplanned outcome which enriches and expands the activity. Having the ability to 'reflect in practice' enables the practitioner to recognise and develop these unplanned, but nevertheless, enriching experiences. Professionalism also manifests itself in very practical considerations: e.g. Risk Assessment Procedures, Criminal Records Bureau/Disclosure ${ }^{18}$ checks (particularly when working with children), awareness of Employers' Liability Insurance, Copyright Law. A practitioner should demonstrate resourcefulness and appropriate initiative. In any engagement experience the engager is an ambassador for the discipline, the $\mathrm{HEI}$ and for the scientific community as a whole.

Finally, public engagement in practice often depends on mutually beneficial arrangements between organisations or between practitioners themselves. A culture of collaboration and interdependence exists between public engagement practitioners which is synergic for design and delivery of activities. This can be at a very simple level for example at the recent Edinburgh International Science Festival, we borrowed a lamp from colleagues and in return we loaned a UV light box. At a different level, I was invited to participate in a project for which a colleague required complementary activities. This presented an opportunity for me to work with a different audience and in turn my colleague was able to fulfil the obligations of her project. These are both fairly pragmatic examples of the 'quid pro quo' culture which exists in public engagement. At a different and more creative level, many public engagement activities and projects are enriched or depend on collaborations between scientists and other institutions and/or areas of expertise: for example museums, galleries, theatres, artists, musicians. The most effective collaborations exist where there is a symbiotic and synergic partnership which extends beyond the life of the project and results in the generation of new and even more innovative engagement ${ }^{19}$.

\section{Principles in Practice}

The principles and ways of practicing have been distilled, by the author of the paper, from over $10+$ years practice in public engagement. To demonstrate these key principles and ways of practicing, two examples of work have been chosen: 1) an in-depth description of how a well-known chemistry demonstration is delivered in schools, and 2) an overview of a grant funded project. These examples demonstrate that the key principles and ways of practicing are relevant to public engagement with science regardless of the scale of the activity.

\section{Example 1}

Adapted from 'Demonstrating the colour changes of indicators using dry-ice ${ }^{20}$.

This was one of the first chemistry demonstrations I learned and it forms the core of many of my demonstration lectures. The demonstration would be one component of a chemistry demonstration lecture delivered in a school classroom for age group 11-14 year olds. Dry-ice is not usually available in 
schools and is a novel means by which to make an acidic solution. A questioning style is used throughout the presentation allowing many opportunities for audience interaction and participation and is an invaluable skill when applied in undergraduate teaching. When visiting a school, the audience consists of both pupils and teacher(s). The aims of the visit are the following:

- To complement the work being carried out in the classroom or to introduce future topics (Feedback from teachers indicates that it is useful if a 'visitor' to the school can reinforce or introduce concepts taught in the classroom e.g. the $\mathrm{pH}$ scale

- To enthuse and pupils about chemistry

- To show the relevance of chemistry in everyday life

- To provide opportunities for knowledge gained during the demonstration to be to be applied to further examples and questions.

- To demonstrate that science is not a collection of unrelated facts.

- For me as a practitioner, to expand my experience and knowledge of working with a particular audience and to gain insights about misconceptions in science and the practice of science.

This particular experiment involves adding a few drops of Universal Indicator (a pHindicator with colour changes across $\mathrm{pH}$ range 1-14) to a volume of water. It is helpful to display the colours of the indicators on a chart. We expect the water to be around $\mathrm{pH} 7$. The colour of the indicator for $\mathrm{pH} 7$ is green, therefore (in most cases!) the water becomes green on addition of the indicator. Then a few drops of vinegar are added to the indicator solution. References can be made to other household and everyday substances which are acids such lemon juice and battery acid. The solution turns from green to red on the addition of a colourless liquid (vinegar). Let's not forget that this is really amazing for someone who has not seen this before. The colour of the solution corresponds to $\sim \mathrm{pH} 2$ on the indicator range confirming that vinegar is an acidic solution. Then a few drops of sodium hydroxide are added (to the same flask). References can be made to Mr Muscle drain cleaner (in which sodium hydroxide is a major component), and other common household alkaline solutions such as shampoo and soap. We are adding a colourless solution to a red solution and the solution turns purple, which corresponds to around $\mathrm{pH} 14$. Now that we know the colours of the indicator for acid, alkali and neutral, we can test whether Dry-ice (solid carbon dioxide) forms an acidic or alkaline solution. Adding the Dry-ice to the purple solution produces a wonderful effect with plumes of fumes (moisture from the atmosphere condensing on the carbon dioxide gas which has sublimed from the solid carbon dioxide) plus we can observe the colour changes across the full range of the Universal Indicator as carbon dioxide in water is an acidic solution and so the colour reverts back to red. The audience can then deduce that carbon dioxide in water is an acidic solution which is why fizzy drinks (which contain carbon dioxide) are not so good for the teeth. The level of explanation of the science can be varied depending on the audience e.g. acids and alkalis, $\mathrm{pH}$ scale, $\mathrm{pH}=-\log _{10}\left[\mathrm{H}_{3} \mathrm{O}^{+}\right]$. Making reference to household substances ensures points of contact with the audience and demonstrates that chemistry and chemicals are not confined to the laboratory. I make the point that everything which is solid, liquid or gas is a chemical, some chemicals are more harmful than others. I also inform the audience that there is a lot of chemistry going in the room in which they are in, and do they know where this chemistry is taking place. It's very gratifying when eleven year old pupils respond by asking variations of: "Is there chemistry going on in our cells?" I extend the demonstration by asking the audience to apply their new knowledge about Universal Indicator and $\mathrm{pH}$ by asking the question: If I was wearing a jumper dyed with Universal Indicator, what colour would it go if I a) stepped out into acid rain? b) washed the jumper with soap powder? The demonstration was extended to include the $\mathrm{pH}$ component of the Global Water Experiment for International Year of Chemistry $2011^{21}$. This involved using two further indicators (bromothymol blue and $m$-cresol purple) to test water samples collected by the school. The experiment was framed in the wider context of availability of clean drinking water in the UK, contrasting this with availability of clean drinking water in other parts of the world and highlighting the tests and procedures required to ensure that water is safe for drinking. The pupils also learned that, regardless of the indicator used in an experiment, the $\mathrm{pH}$ scale is used universally to measure the acidity of a substance. The 'performance' of the demonstration can be amended, adapted and extended to meet audience requirements.

\section{Demonstrating key principles:}

The science is accurate at a level appropriate for the audience. References are made to household substances which are familiar to the audience in order to establish common points of interest and knowledge before introducing new concepts. By providing references to everyday substances, this enables pupils to recognise that chemistry and chemicals are part of their everyday life and not something which is only experienced in the laboratory during chemistry lessons. Care is taken to give balanced information: for example explaining that chemicals can be essential, beneficial or harmful to humans and to the environment; and this applies to man-made as well as naturally occurring chemicals. By enabling the pupils to apply the knowledge gained during the demonstration to other example (the Universal Indicator jumper), this illustrates the application of a fundamental concept in a different context and allows the pupils to test their understanding of the concept and extend their understanding.

Therefore the key principles of accuracy of science, point of common interest, clarity of purpose (defined in the aims) neutrality, and a net gain or insight are satisfied. As a bonus I acquired a new name during one school visit: 'the Ice-Lady'!

Demonstrating 'Ways of Practicing':

The aims are clearly defined and provide guidance in the design and delivery of the activity. By reflecting on the audience reactions, perceptions and understanding during and after the visit, the demonstration can be refined and adapted as necessary. The 'Dry-ice demonstration is one component of a demonstration lecture which has to be flexible enough to expand or contract depending on the class time available which can vary between school by as much as 30 minutes. It is unrealistic to expect schools to reschedule class times to suit one visitor. Risk Assessments are of course carried out and provided to the school. The demonstration has evolved and has been adapted for different contexts including a hands-on version as a component of a workshop. It was possible to include the $\mathrm{pH}$ component of the IYC Global Water Experiment to give a wider context to the introduction of $\mathrm{pH}$ 
and I requested that the school collect water samples. Whilst we do not charge for workshops and demonstrations, we do insist that schools participate in the evaluation of the activity during the visit and by providing comments after the event. Reflective practice, professionalism, flexibility and quid pro quo are all demonstrated in this example.

\section{Example 2}

The second example is based on the project 'Superbugs' - a Challenge for $21^{\text {st }}$ Century Scientists ${ }^{22}$ which was a Wellcome Trust funded People Award. This was a collaborative project between researchers and public engagement practitioners. The researchers were exploring the pathology of a superbug (Burkholderia cenocepacia) which affects individuals with Cystic Fibrosis (CF).

The aim of the project was to raise awareness of superbugs and of the multidisciplinary approaches required to combat them. This was achieved by delivering combined hands-on/ discussion workshops for school pupils together with public events at the Edinburgh International Science Festival, in the National Museums Scotland and at the Edinburgh Festival Fringe. The workshops consisted of hands-on activities exploring the following: effective hand-washing, structure and functionality of bacteria, identification, diagnosis and treatment of superbug infections. The discussion activities explored the implications of superbugs in everyday life and in the lives of individuals who have CF.

During the project the following took place: over 20 schools participated in workshops; 5 days of activities were delivered at the Edinburgh International Science Festival (average of 800 visitors per day $\sim 200$ of whom spent 20 minutes or more at the activity); 3 days of activities at the National Museums Scotland (around 100 visitors, per day who spent 20 minutes or more at the activities); 2 days of activities at the Edinburgh Festival Fringe (around 70 visitors per day. The audiences for the public events were often children accompanied by relatives/carers (often grandparents). The workshop resources have subsequently been modified so that they can be delivered by teachers in schools. CDs of resources including video footage of bad hand hygiene practice were distributed to all schools who participated in activities and to other schools visited by University of Edinburgh as part of their outreach programme.

The key learning outcomes designed for the project were as follows:

- importance of hand-hygiene

- causes of antibiotic resistance of bacteria

- an understanding of the multidisciplinary approach required to address the issue of superbugs

These were successfully communicated and discussed with participants (indicated by the project evaluation data). The hands-on workshops were well received in schools (with pupils and teachers) and complemented key learning outcomes in science, health and well-being and social studies in the Curriculum for Excellence ${ }^{23}$ (launched in Scottish schools in 2010). The project provided valuable experience and development in public engagement practice for post-graduate students from the School of Chemistry and Biological Sciences and for the researchers involved in the project.
Demonstrating key principles:

The issue of superbugs and hospital hygiene is constantly in the news and provides a very topical and practical focus for both hands-on and discussion activities. The 'Superbugs' project built on the success of the 'Biomedical Horizons' 24 project (Wellcome People Award 2005). One of the hands-on activities which was very popular in Biomedical Horizons was the use of a light box which illuminates areas of the hand which have not been washed thoroughly. In 'Biomedical Horizons' we found that by actively involving participants in hands-on activities early on in the workshop had the effect of generating a more relaxed and inclusive discussion about the science and about issues raised by the workshop. The hand-washing component of 'Superbugs' was a simple yet effective activity to engage participants immediately and which provoked discussion about the transfer of bacteria.

Another activity involved building a superbug from its component parts e.g. cell membrane, cell wall, ribosomes, plasmids, flagella. This enabled a discussion about how researchers might approach the development of potential antibiotic therapies i.e. by studying the formation and function of components of the cells of superbugs, researchers are able to identify potential weaknesses which could be exploited for the development of therapies. For example in the case of the superbug Burkholderia cenocepacia the weakness appeared to be the chemistry of the outer cell wall synthesis ${ }^{25}$. Thus we were able to communicate the research work accurately but at a level appropriate for the audience and demonstrate that chemists and microbiologists were involved in the research.

A second activity involved the pupils following evidence to diagnose and prescribe treatment for the infections of fictional patients. This was originally designed for more senior pupils (16-18 year olds) and adults but proved so popular that a simpler version was designed for younger participants. The pupils commented that they enjoyed following through the procedure of diagnosis and treatment, using information they had learned, and applying it in a fictional case. The workshops highlighted the fact that it was not only medical doctors who were involved in this work but chemists and microbiologists ranging from eminent researchers to laboratory technicians. The PhD student helpers valued the experience in communicating and discussing areas of science with school level pupils and discovering pupil perceptions of scientists.

\section{Demonstrating 'Ways of Practicing':}

The objectives for the project together with the public engagement methodologies are defined in funding proposal s which ensures that much of the strategic planning is completed before the funding is awarded and enables the design of an effective evaluation strategy for the project at the outset.

The activities were trialled then amended after feedback from a teacher with whom we worked in partnership. The activities were designed to be flexible and able to be adapted and updated depending on the circumstances and audiences to which they were being delivered. PhD students helpers and laboratory technicians from the CF project were given both generic and workshop specific science communication and were encouraged to, and indeed did, reflect on their delivery of workshops as evidenced by their feedback in evaluation. By working closely with researchers, aspects of their research were accurately disseminated to an audience beyond the academic community. 


\section{Conclusions}

Public engagement with science is a practice which enables, extends and enriches the generation and particularly the dissemination of knowledge to various publics within and beyond academia.

Public engagement is a 'way of thinking' about science i.e. thinking about the science from the overview, from the wider context, and then identifying common points of interest/ knowledge/experience with an audience. The key principles for public engagement with science are the following:

- Accuracy of science

- The 'audience perspective' as the starting point

- Clarity of purpose

- Neutrality

- Enriching experience i.e. there should be a 'net gain' for all participants as a result of the public engagement.

Public engagement with science is developing its own 'Community of Practice' with reflective practice, professionalism, flexibility and quid pro quo being the cornerstones of effective practice.

\section{References}

1. HEFCE Publication 2006 Beacons for Public Engagement: invitation to apply for funds, 2006/49. Viewed online at $<w w w . h e f c e . a c . u k / p u b s / h e f c e / 2006 / 06 \_49 />$ [Accessed 19 December 2006].

2. NCCPE. National Co-ordinating Centre for Public Engagement: About Us. Viewed online at <www.publicengagement.ac.uk/about> [Last accessed 21 May 2011].

3. Research Councils UK. Pathways to Impact: expectations and policies. Viewed online at $<$ www.rcuk.ac.uk/kei/impacts/Pages/ expectationpolicies.aspx> [Last accessed 21 May 2011].

4. NCCPE. How to Support Public Engagement. Viewed online at $<$ www.publicengagement.ac.uk/support> [Last accessed 21 May 2011].

5. McTaggart, R. (1989) 16 Tenets of Participatory Action Research. Viewed online at http://www.caledonia.org.uk/ par.htm. [Last accessed 21 May 2011].

6. NCCPE: What purposes can public engagement serve? Viewed online at $<$ www.publicengagement.ac.uk/what/purposes-ofengagement>

[Last accessed 21 May 2011].

7. Stevenson, E. (1999). Pyrolitic Syntheses of Fused Bridgehead Nitrogen Heterocycles. PhD thesis.

8. Camp, P. Research Interests. Viewed online at <www.chem.ed.ac.uk/staff/academic/camp.htm> [Last accessed 18 June 2011].

9. REF 2014 Research Excellence Framework. Viewed online at $<$ www.hefce.ac.uk/research/ref/> [Last accessed 21 May 2011].

10. Weitkamp, E., Smith, D., Featherstone, H. Selenia. Viewed online at $<w w w . s c i e n c e c o m i c s . u w e . a c . u k / c o m i c s . p h p>$ [Last accessed 19 June 2011].
11. du Sautoy, M. About Maths in the City. Viewed online at <www.mathsinthecity.com/info/about-maths- city> [Last accessed 21 May 2011].

12. NCCPE. What purposes can public engagement serve? Viewed online at $<w w w$.publicengagement.ac.uk/what/purposes-ofengagement> [Last accessed 19 June 2011].

13. Don, M. (2011) Monty Don's Italian Gardens <www.bbc.co.uk/programmes/>

14. Barfoot, J. (2009) Science Communication elective, School of Biomedical Sciences, University of Edinburgh

15. Stevenson, E. Personal reflections on the 'quality in public engagement workshops' in Edinburgh and Manchester (Beacons for Public Engagement). <elizstevenson.wordpress.com/>

16. Schön, D. (1983) The Reflective Practitioner, How Professionals think in action, London: Temple Smith

17. Courses in Chemistry, Quality Assurance Agency for Higher Education (1998) General Guidelines for the Academic Review of Bachelors Honours Degree

18. Disclosures.co.uk. About. Viewed online at <www.disclosures.co.uk/google adwords/> [Last accessed 19 June 2011].

19. EPSRC. A computer that tells jokes, you're having a laugh. Viewed online at

$<w w w . e p s r c . a c . u k / n e w s e v e n t s / n e w s / 2009>$ [Last accessed 21 May 2011].

20. The Royal Society of Chemistry. (1995) Classic Chemistry Demonstrations, Education Division, Royal Society of Chemistry.

21. International Year of Chemistry 2011. The experiments. Viewed online at <water.chemistry2011.org//web/iyc/24> [Last accessed 19 June 2011].

22. Wellcome Trust. People award summaries October 2008-September 2009. Viewed online at $<$ www.wellcome.ac.uk/Funding/Public-engagement/ Funded-projects/Awards-made/All-awards- made/ index.htm> [Last accessed 19 June 2011].

23. Learning Teaching Scotland. What is Curriculum for Excellence? Viewed online at $<w w w$.Itscotland.org.uk/understandingthecurriculum/ whatiscurriculumforexcellence/index.asp> [Last accessed 19 June, 2011]

24. Biomedical Horizons $<$ horizons.bio.ed.ac.uk/> [Last accessed 19 June, 2011]

25. UK Cystic Fibrosis Microbiology Consortium: Research with the Microbiology Consortium, Programme 4 Novel antimicrobial agents. Viewed online at $<$ www.cfmicrobiology.org.uk/research/> [Last accessed 19 June 2011]. 